COLABORACIÓN ESPECIAL

\title{
RELACIÓN ENTRE LA ASISTENCIA A GUARDERÍAS Y ENFERMEDAD INFECCIOSA AGUDA EN LA INFANCIA. UNA REVISIÓN SISTEMÁTICA
}

\author{
Carlos Ochoa Sangrador (1), Ma Verísima Barajas Sánchez (1) y Beatriz Muñoz Martín (2) \\ Servicio de Pediatría. Hospital Virgen de la Concha. Zamora. \\ Biblioteca. Hospital Virgen de la Concha. Zamora.
}

\section{RESUMEN}

La asistencia al jardín de infancia es considerada un factor de riesgo de enfermedad aguda en la primera infancia, sin embargo los trabajos disponibles no permiten cuantificar de forma integrada dicho riesgo. Realizamos una revisión sistemática de ensayos clínicos y estudios de cohortes en los que se hayan estudiado los efectos de la asistencia de día en guarderías sobre la salud infantil en las bases de datos de la Colaboración Cochrane, PubMed e Índice Médico Español, sin límites de idioma ni de tiempo, completando con análisis de citas y una búsqueda suplementaria en EMBASE. Valoramos la calidad metodológica mediante criterios personalizados. Calculamos medidas de efecto resumen (riesgos relativos, razones de densidades de incidencia y diferencias de medias ponderadas), con sus intervalos de confianza, asumiendo modelos de efectos aleatorios. Hemos observado un significativo aumento de riesgo consistente a lo largo del tiempo y entre diferentes entornos sociales y geográficos. Considerando los trabajos con mayor rigor metodológico y con estimadores de efecto ajustados, la asistencia a guardería se asoció a un incremento de riesgo de infección respiratoria alta $(\mathrm{RR}=1,88)$, otitis media aguda $(\mathrm{RR}=1,58)$, otitis media con derrame $(\mathrm{RR}=2,43)$, infecciones respiratorias bajas (globalmente $\mathrm{RR}=2,10$; neumonías $R R=1,70$; bronquiolitis $R R=1,80$; bronquitis $R R=2,10$ ) y gastroenteritis agudas $(\mathrm{RR}=1,40)$. La asistencia a guardería podría ser la responsable de entre un 33\% y un 50\% de los episodios de infección respiratoria y gastroenteritis en la población expuesta. Como conclusión podemos decir que el riesgo sobre la salud infantil de la asistencia a guardería es discreto pero de un gran impacto. Esta información tiene importantes implicaciones para la investigación, la práctica clínica, las autoridades sanitarias y la sociedad.

Palabras clave: Guardería. Factores de riesgo. Enfermedad aguda. Infección respiratoria. Gastroenteritis.

Correspondencia:

Carlos Ochoa Sangrador

Unidad de Investigación

Hospital Virgen de la Concha.

Avda. Requejo 35

49029 Zamora.

Correo electrónico: cochoas@meditex.es
ABSTRACT

\section{Relationship between Child Day-Care Attendance and Acute Infectious Disease. A Systematic Review}

Child day-care attendance is considered to be an acute early childhood disease risk factor, the studies available however not affording the possibility of fully quantifying this risk. A systematic review of clinical trials and cohort studies was conducted, in which the effects child day-care attendance had on the health of young children based on the Cochrane Collaboration, PubMed and Spanish Medical Index databases, without any time or language-related limits, were analyzed and rounded out with analyses of referenced works and an additional EMBASE search. The methodological quality was evaluated by means of personalized criteria. Pooling measures (relative risks, incidence density ratios and weighted mean differences) were calculated with their confidence intervals, assuming random effects models. A significant increase was found to exist of a risk consistent over time and among different social and geographical environments. Considering the most methodologicallystringent studies with adjusted effect estimates, child day-care attendance was related to an increased risk of upper respiratory tract infection $(\mathrm{RR}=1,88)$, acute otitis media ( $R R=1,58)$, otitis media with fluid draining $(R R=2,43)$, lower respiratory tract infections (overall $R R=210$; acute pneumonia $\mathrm{RR}=1.70$; broncholitis $\mathrm{RR}=1,80$; bronchitis $\mathrm{RR}=2,10)$ and gastroenteritis $(\mathrm{RR}=1,40)$. Child day-care attendance could be responsible for $33 \%-50 \%$ of the episodes of respiratory infection and gastroenteritis among the exposed population. In conclusion, it can be said that the risk for childhood health attributable to the child day-care attendance is discreet but of high-impact. This information has some major implications for research, clinical practice, healthcare authorities and society as a whole.

Keywords: Children. Child day care centers. Risk factors. Acute disease. Respiratory tract infection. Gastroenteritis. 


\section{INTRODUCCIÓN}

En los últimos años se viene manteniendo un debate social sobre cómo, dónde y quién debe cuidar a los niños pequeños. La prestación de cuidados durante el día fuera del hogar varía ampliamente de unos países a otros. Entre 1990 y 1994 los países europeos con mayor cobertura escolar en menores de 3 años eran Alemania oriental (50\%), Dinamarca (48\%) y Suecia (33\%), encontrándose entonces España muy por debajo con un $2 \%{ }^{1}$. En nuestro país estamos asistiendo a un aumento progresivo de la demanda y creación de plazas de guardería, con importantes diferencias entre regiones. Este incremento es consecuencia de distintos factores de índole sociolaboral, como es el trabajo de la madre fuera del hogar, tanto de la población nacional como de la inmigrante, o el aumento de las familias monoparentales. Actualmente sólo un 13,9\% de los niños menores de 3 años están escolarizados en centros de educación infantil ${ }^{2}$, mientras que un $20,7 \%$ de la población activa declara utilizar servicios especializados de guardería para el cuidado de sus hijos ${ }^{3}$.

Es opinión generalizada entre padres y profesionales que la escolarización precoz origina un aumento en la incidencia de enfermedad aguda infantil. Podemos encontrar en la literatura múltiples referencias a este fenómeno, pero cuesta localizar estudios que realicen una valoración integrada del problema, existiendo en dichos trabajos una gran heterogeneidad en los objetivos perseguidos, las poblaciones seleccionadas, los diseños utilizados y los análisis empleados, lo que supone un obstáculo para la valoración e interpretación de la evidencia disponible $e^{4}$. Por otra parte, en los últimos años diferentes estudios están explorando la posibilidad de que la exposición precoz a agentes infecciosos pueda ocasionar cierta protección frente a enfermedades de base o implicación inmune en la edad adulta. No obstante, el papel de la asistencia a guarderías en estas enfermedades está todavía por aclarar ${ }^{5-7}$.

Una revisión sistemática de 1998, que incluía 8 ensayos controlados y aleatorizados (ECA) realizados en Estados Unidos de América (EEUU), mostró que para población socialmente desfavorecida o con antecedente de prematuridad la asistencia a guardería presentaba efectos beneficiosos sobre el desarrollo conductual en la infancia, el éxito escolar y patrones económicos, laborales y sociales en la vida adulta $^{8}$. Sin embargo, sólo en uno de los ECA se consideraron resultados relacionados con la salud de los niños, mostrando que una estrategia de intervención precoz (con asistencia a centros de día al menos 2 años) no incrementaba el riesgo de recibir asistencia hospitalaria, pero sí aumentaba discretamente el número de consultas ambulatorias por enfermedad aguda ${ }^{9}$.

Los ECA son considerados el patrón de referencia en la evaluación de intervenciones sanitarias ${ }^{10}$, ya que otros tipos de estudio tienden a producir estimaciones sesgadas de su eficacia ${ }^{11}$. Sin embargo, el estudio de intervenciones sociales mediante ECA resulta problemático, tanto por la existencia de dificultades éticas y logísticas para su ejecución como por las limitaciones que las restricciones metodológicas originan a la hora de generalizar sus resultados. Por lo tanto resulta comprensible que la mayor parte de la literatura publicada con respecto a los efectos sobre la salud de la asistencia a guarderías se sustente en estudios observacionales. Como muestra de ello baste mencionar dos revisiones sistemáticas publicadas en 1996 y $1999^{12,13}$, las cuales exploraban la asociación entre la incidencia de otitis media aguda y la asistencia a guardería, y que sólo incluyeron estudios de cohortes, casos control y estudios transversales.

Pero no todos los estudios observacionales aportan la misma validez en investi- 
gación etiológica; sólo los diseños con direccionalidad anterógrada (estudios de cohortes) permiten un buen control de la heterogeneidad inicial de las cohortes y una vigilancia de la secuencia temporal de los acontecimientos. De hecho, la utilización de estudios de direccionalidad retrógrada (estudios de casos y controles) puede dar lugar a resultados sesgados que ocasionan hipótesis llamativas ${ }^{5}$. Por estos motivos, en ausencia de ensayos clínicos representativos parece sensato utilizar los resultados de estudios de cohortes para identificar el impacto sobre la salud de la asistencia a guarderías en la primera infancia.

\section{MATERIAL Y MÉTODOS}

Realizamos una revisión sistemática de la literatura para evaluar los efectos de la asistencia de día en guarderías sobre la salud infantil.

Tipos de estudios: ECA, tanto aleatorizados como cuasi-aleatorizados, y estudios de cohortes, tanto prospectivos como retrospectivos. No hubo restricciones de idioma.

Tipos de participantes: Estudios que incluyeran población expuesta a guarderías antes de los 3 años de edad con seguimiento durante los años de asistencia o posteriores.

Tipos de comparación: Comparación entre niños atendidos en guarderías ("daycare centers", "schools nursery"; asistencia fuera del hogar por personal ajeno al entorno familiar en centros con al menos siete niños) y otras formas de cuidado ("home care"; niños atendidos en su domicilio o por personas del entorno familiar). Los casos clasificados por su atención en domicilios ajenos con grupos reducidos de niños ("family day care") fueron analizados como una categoría aparte cuando esta información estaba desagregada. Por lo tanto, se consideraron 3 niveles de exposición que correspondían a: I) asistencia en casa ("home care"), II) asistencia en domicilios ajenos en grupos reducidos ("family day care"), y III) asistencia en guardería.

Tipos de medidas de resultado: Efectos sobre la salud infantil: ingresos hospitalarios, consultas médicas, lesiones, infecciones respiratorias agudas, infecciones respiratorias de vías altas o bajas, rinitis, catarro común, crup, otitis media aguda, otitis media con derrame, sibilantes, asma, bronquitis, bronquiolitis, neumonía, diarrea-gastroenteritis y otras infecciones (citomegalovirus, portador de neumococo resistente). Se excluyeron expresamente las medidas de desarrollo intelectual o conductual.

Estrategia de búsqueda para la identificación de los estudios: Realizamos búsquedas bibliográficas en las bases de datos de la Cochrane Library (http: // www.update-software.com/clibplus/clibpluslogon.htm) y de la National Library of Medicine de los Estados Unidos (http: // www.ncbi.nlm.nih.gov/entrez/query.fcgi? $\mathrm{CMD}=$ search $\& \mathrm{DB}=$ pubmed), sin límites de idioma ni fecha (actualizadas hasta el 17 de junio de 2006). La búsqueda se completó mediante la revisión manual de las citas bibliográficas y una búsqueda en el Índice Médico Español con el término "guardería".

En la Cochrane Library buscamos revisiones sistemáticas y ensayos clínicos empleando los descriptores: [child and (daycare or (nursery next school))].

En PubMed buscamos revisiones sistemáticas con descriptores equivalentes y un filtro metodológico específico: (( ("Child Day Care Centers"[MeSH] OR (child AND (day care OR daycare))) OR ("Schools, Nursery"[MeSH] OR Nursery School*)) OR ("family day care" OR "day care homes")) AND systematic[sb]. Para buscar 
ECA empleamos un filtro metodológico sensible: ((("Child Day Care Centers" [MeSH] OR (child AND (day care OR daycare))) OR ("Schools, Nursery" [MeSH] OR Nursery School*)) OR ("family day care" OR "day care homes")) AND ((clinical[Title/Abstract] AND trial [Title/Abstract]) OR clinical trials[MeSH Terms] OR clinical trial[Publication Type] OR random*[Title/Abstract] OR random allocation[MeSH Terms] OR therapeutic use[MeSH Subheading]). Finalmente para localizar estudios de cohortes usamos una estrategia que contenía un filtro metodológico específico: (( ("Child Day Care Centers" [MeSH] OR (child AND (day care OR daycare))) OR ("Schools, Nursery" [MeSH] OR Nursery School*)) OR ("family day care" OR "day care homes")) AND ((relative[Title/Abstract] AND risk* [Title/Abstract]) OR (relative risk[Text Word]) OR risks[Text Word] OR cohort studies[MeSH: noexp] OR (cohort[Title/Abstract] AND stud*[Title/Abstract]))

Para controlar la sensibilidad de la estrategia de búsqueda utilizada, fundamentalmente de las bases de datos seleccionadas, se realizó a posteriori una búsqueda de ensayos clínicos y estudios de cohortes en EMBASE, con descriptores equivalentes a los empleados en PubMed.

Revisión de trabajos: Dos evaluadores leyeron los títulos y resúmenes de los artículos localizados por las búsquedas para descartar estudios que no cumplieran criterios de inclusión. Una vez recuperados los artículos relevantes, ambos evaluadores analizaron el texto completo, para poder juzgar el cumplimiento de los criterios de inclusión en la revisión. Toda discordancia en la evaluación se resolvió por consenso.

En ausencia de criterios de calidad estandarizados para revisiones sistemáticas de estudios observacionales, utilizamos siete parámetros de calidad que fueron categorizados para cada estudio: A) homoge- neidad basal de los grupos de estudio (ausencia de sesgos de selección) y representatividad de la muestra; B) medición válida y homogénea del factor de exposición (B1) y el efecto (B2); C) seguimiento completo y no diferencial; D) control de la relación temporal de los acontecimientos (D1) y de la relación dosis-respuesta (tiempo de exposición; D2); y E) control de factores de confusión mediante estratificación o análisis multivariante. Estos criterios se encuentran recogidos en distintas guías de valoración de estudios epidemiológicos ${ }^{14}$. No asignamos puntuación a los criterios de calidad, considerándose sólo cualitativamente a la hora de la inclusión de los estudios o la realización de análisis de sensibilidad.

Recopilamos los resultados expresados en cada trabajo como recuentos de eventos o sus medias y desviaciones típicas, por separado para los grupos guardería y control, así como los detallados en forma de medidas de riesgo (riesgos relativos, odds ratios y razones de densidades de incidencia) con sus respectivos intervalos de confianza. A partir de los intervalos de confianza se calcularon los errores típicos. Para poder agrupar riesgos se convirtieron las odds ratios en riesgos relativos, cuando aquéllas se presentaban ajustadas o no se podían calcular los riesgos a partir de recuentos, empleando para su conversión el riesgo existente en el grupo control ${ }^{15}$.

Cuando se disponía de más de un estudio para cada medida de efecto y la heterogeneidad de su metodología y resultados lo permitía, se calcularon los estimadores ponderados resumen (diferencias de medias ponderadas, riesgos relativos y razones de densidades de incidencia) utilizando modelos de efectos aleatorios, por ofrecer los resultados más conservadores. Todos los cálculos se realizaron con el programa RevMan (v.4.2.7), en el que se introdujeron los recuentos y/o los estimadores de riesgo de cada trabajo. Se realizó 
una selección de estudios con mayor rigor metodológico (estudios de temporalidad concurrente, ECA y estudios de cohortes prospectivos, con adecuado seguimiento y con ajuste multivariante), que fueron considerados para un análisis selectivo, del que se obtuvieron nuevos estimadores resumen (análisis de sensibilidad).

\section{RESULTADOS}

La búsqueda realizada en la Cochrane Library recuperó 83 documentos (10 revisiones sistemáticas y 71 ensayos clínicos), de los que sólo estaban relacionados una revisión sistemática ${ }^{8}$ y un $\mathrm{ECA}^{9}$. En PubMed la búsqueda de revisiones sistemáticas localizó 62 citas de las que sólo 5 estaban relacionadas (correspondientes a 4 trabajos), la de ensayos clínicos recuperó 1.057 citas, de las que sólo 29 estaban relacionadas (incluyendo 1 revisión sistemática) y la de estudios de cohortes 304 citas de las que sólo 49 estaban relacionadas (incluyendo 1 revisión sistemática). Combinando los resultados de las búsquedas anteriores se obtuvieron 68 referencias que cumplieran los criterios de inclusión (tras descartar duplicidades). En la búsqueda realizada en el Índice Médico Español se encontraron 2 estudios previamente ya identificados.

Tras una revisión de las referencias de los artículos recuperados se amplió la revisión con 53 artículos más, de los cuales 24 cumplían criterios de inclusión. Finalmente 92 artículos, que correspondían a 84 estudios, fueron considerados para su análisis detallado.

Las revisiones sistemáticas (4) y los estudios de casos y controles (6) fueron revisados pero no se consideraron para su valoración cuantitativa. Se excluyeron 32 trabajos, 6 por ser estudios de casos y controles ${ }^{6,16-20}, 2$ por ser nuevos análisis de estudios ya incluidos ${ }^{21,22}, 10$ por ser estu- dios de cohorte única sin grupo control ${ }^{23-}$ ${ }^{32}, 7$ por analizar exclusivamente parámetros de desarrollo o medidas no relacionadas con enfermedad aguda ${ }^{33-39}, 4$ por ser revisiones sistemáticas ${ }^{5,8,12,13}$ y 3 por estudiar muestras de pacientes inadecuadas $^{40-42}$.

En la búsqueda realizada a posteriori en la base de datos EMBASE se localizaron 382 citas, de las que solamente 7 aportaban información suplementaria previamente no identificada. Entre estas citas no había ningún ensayo clínico y sólo 3 correspondían a estudios de cohortes sobre factores de riesgo de otitis media aguda ${ }^{43-45}$, de los que sólo 1 se centraba en el análisis de la exposición a guardería ${ }^{44}$.

Aportaron información válida con estimadores de efecto cuantificables 52 estudios (tabla 1): 2 ensayos clínicos, 32 estudios de cohortes prospectivos y 18 estudios de cohortes retrospectivos (13 de éstos eran estudios transversales con recopilación histórica). Los estudios fueron realizados en 15 países, fundamentalmente en Estados Unidos (21) y países nórdicos (Finlandia 6, Suecia 6, Noruega 3); del resto 11 se hicieron en otros países europeos (1 en España), 2 en Canadá, 1 en México, 1 en Colombia y otro recopiló una muestra multinacional.

En total se estudió a 127.088 niños. El tamaño de las muestras osciló entre 63 y 20.016 (mediana 932,5). La duración del seguimiento varió desde dos semanas hasta 13 años (mediana 1,5 años).

Los métodos de selección de las cohortes más habituales en los estudios prospectivos fueron la selección de muestras de recién nacidos en un centro o área sanitaria, o muestras de niños de revisiones programadas en centros de salud, clasificados en distintas cohortes en función de la exposición a guardería; otras veces se utilizaron muestras de niños atendidos en guarderías 
Tabla 1

Descripción y criterios de calidad de los estudios incluidos en la revisión

\begin{tabular}{|c|c|c|c|c|c|c|c|c|c|c|c|}
\hline \multirow[b]{2}{*}{ Autor ${ }^{\text {cita }}$. Año. } & \multirow[b]{2}{*}{ País } & \multirow[b]{2}{*}{ Diseño } & \multirow[b]{2}{*}{ Tiempo } & \multicolumn{8}{|c|}{ Criterios de Calidad } \\
\hline & & & & $\mathbf{N}^{\mathbf{o}}$ & $\mathbf{A}$ & B1 & B2 & C & D1 & D2 & $\mathbf{E}$ \\
\hline Alexander ${ }^{60} 1990$ & EEUU & $\mathrm{ET}(\mathrm{CR})$ & & 4.845 & NA & No & $\mathrm{Si}$ & NA & $\mathrm{Si}$ & No & $\mathrm{Si}$ \\
\hline Alho ${ }^{61,62} 1990$ & Finlandia & $\mathrm{ECP} *$ & 2 a. & 2.512 & No & $\mathrm{Si}$ & $\mathrm{Si}$ & $\mathrm{Si}$ & $\mathrm{Si}$ & No & $\mathrm{Si}$ \\
\hline Ball $^{56,57} 2000$ & EEUU & $\mathrm{ECP*}$ & $13 \mathrm{a}$. & 1.246 & No & $\mathrm{Si}$ & $\mathrm{Si}$ & $\mathrm{Si}$ & $\mathrm{Si}$ & $\mathrm{Si}$ & $\mathrm{Si}$ \\
\hline Barros $^{63} 2003$ & Portugal & ECR & $3 \mathrm{a}$. & 292 & No & $\mathrm{Si}$ & $\mathrm{Si}$ & NA & No & $\mathrm{Si}$ & $\mathrm{Si}$ \\
\hline Bartlett ${ }^{64} 1985$ & EEUU & $\mathrm{ECP} *$ & 2 a. & 561 & No & $\mathrm{Si}$ & No & NV & $\mathrm{Si}$ & $\mathrm{Si}$ & $\mathrm{Si}$ \\
\hline Bell ${ }^{65} 1989$ & EEUU & ECP* & $7 \mathrm{~m}$. & 843 & No & $\mathrm{Si}$ & $\mathrm{Si}$ & NV & $\mathrm{Si}$ & $\mathrm{Si}$ & $\mathrm{Si}$ \\
\hline Celedónn ${ }^{51-53} 1999$ & EEUU & $\mathrm{ECP} *$ & $6 \mathrm{a}$. & 498 & No & No & $\mathrm{Si}$ & $\mathrm{Si}$ & $\mathrm{Si}$ & No & $\mathrm{Si}$ \\
\hline Collet $^{48} 1991$ & Francia & ECR & $2 \mathrm{~m}$. & 1.624 & No & $\mathrm{Si}$ & No & $\mathrm{Si}$ & $\mathrm{Si}$ & No & $\mathrm{Si}$ \\
\hline Collet $^{66} 1994$ & Francia & $\mathrm{ECP} *$ & $8,5 \mathrm{~m}$ & 1.037 & No & $\mathrm{Si}$ & $\mathrm{Si}$ & $\mathrm{Si}$ & $\mathrm{Si}$ & No & $\mathrm{Si}$ \\
\hline Demissie $^{67} 1998$ & Canadá & $\mathrm{ET}(\mathrm{CR})$ & & 1.111 & NA & No & $\mathrm{Si}$ & No & No & No & $\mathrm{Si}$ \\
\hline Doyle $^{68} 1976$ & Canadá & ECP & $2 \mathrm{~m}$. & 63 & $\mathrm{Si}$ & $\mathrm{Si}$ & $\mathrm{Si}$ & NV & $\mathrm{Si}$ & No & No \\
\hline Engel $^{69,70} 1999$ & Holanda & $\mathrm{ECP}$ & $2 \mathrm{a}$. & 250 & No & $\mathrm{Si}$ & $\mathrm{Si}$ & No & $\mathrm{Si}$ & No & $\mathrm{Si}$ \\
\hline Fleming $^{71} 1987$ & EEUU & $\mathrm{ET}(\mathrm{CR})$ & & 449 & NA & No & $\mathrm{Si}$ & NA & $\mathrm{Si}$ & No & $\mathrm{Si}$ \\
\hline Flores $^{72} 1999$ & México & $\mathrm{ECP}^{*}$ & $1 \mathrm{a}$. & 300 & No & $\mathrm{Si}$ & $\mathrm{Si}$ & $\mathrm{Si}$ & $\mathrm{Si}$ & No & $\mathrm{Si}$ \\
\hline Forssell $^{73} 2001$ & Suecia & ECR & 1 a. & 218 & NA & $\mathrm{Si}$ & No & NA & No & No & $\mathrm{Si}$ \\
\hline Gardner $^{74} 1984$ & EEUU & $\mathrm{ECP}$ & $1 \mathrm{a}$. & 92 & No & $\mathrm{Si}$ & $\mathrm{Si}$ & NV & $\mathrm{Si}$ & No & No \\
\hline Hardy $^{75} 1988$ & EEUU & $\mathrm{ET}(\mathrm{CR})$ & & 5.818 & NA & No & No & NA & No & $\mathrm{Si}$ & $\mathrm{Si}$ \\
\hline Harsten $^{76} 1989$ & Suecia & $\mathrm{ECP}$ & 3 a. & 122 & NA & $\mathrm{Si}$ & $\mathrm{Si}$ & $\mathrm{Si}$ & $\mathrm{Si}$ & No & No \\
\hline Hillis $^{77} 1992$ & Colombia & $\mathrm{ECP}^{*}$ & $5 \mathrm{~m}$. & 489 & $\mathrm{Si}$ & $\mathrm{Si}$ & $\mathrm{Si}$ & $\mathrm{Si}$ & $\mathrm{Si}$ & $\mathrm{Si}$ & $\mathrm{Si}$ \\
\hline Holberg $^{78} 1993$ & EEUU & $\mathrm{ECP} *$ & $3 \mathrm{a}$. & 1.055 & No & $\mathrm{Si}$ & $\mathrm{Si}$ & $\mathrm{Si}$ & $\mathrm{Si}$ & $\mathrm{Si}$ & $\mathrm{Si}$ \\
\hline Hurwitz $^{79} 1991$ & EEUU & $\mathrm{ET}(\mathrm{CR})$ & & 2.137 & NA & No & $\mathrm{Si}$ & NA & $\mathrm{Si}$ & $\mathrm{Si}$ & $\mathrm{Si}$ \\
\hline Kero $^{80} 1987$ & Finlandia & $\mathrm{ECP}^{*}$ & $1 \mathrm{a}$. & 5.356 & No & $\mathrm{Si}$ & $\mathrm{Si}$ & $\mathrm{Si}$ & $\mathrm{Si}$ & No & $\mathrm{Si}$ \\
\hline Koch $^{81} 2003$ & Dinamarca & $\mathrm{ECP} *$ & 2 a. & 288 & No & $\mathrm{Si}$ & $\mathrm{Si}$ & $\mathrm{Si}$ & $\mathrm{Si}$ & No & $\mathrm{Si}$ \\
\hline Koopman $^{82} 2001$ & Holanda & $\mathrm{ECP} *$ & 1 a. & 4.146 & No & $\mathrm{Si}$ & $\mathrm{Si}$ & $\mathrm{Si}$ & $\mathrm{Si}$ & No & $\mathrm{Si}$ \\
\hline Kramer $^{83} 1999$ & Alemania & $\mathrm{ET}(\mathrm{CR})$ & & 2.471 & NA & No & $\mathrm{Si}$ & NA & No & $\mathrm{Si}$ & $\mathrm{Si}$ \\
\hline Leino $^{84} 2001$ & Finlandia & $\mathrm{ECP}^{*}$ & $2 \mathrm{a}$. & 100 & No & $\mathrm{Si}$ & $\mathrm{Si}$ & $\mathrm{NV}$ & $\mathrm{Si}$ & No & $\mathrm{Si}$ \\
\hline Louhiala $^{85,86} 1995$ & Finlandia & $\mathrm{ET}(\mathrm{CR})$ & & 2.568 & NA & No & $\mathrm{Si}$ & NA & No & No & $\mathrm{Si}$ \\
\hline $\mathrm{Lu}^{87} 2004$ & EEUU & $\mathrm{ECP}^{*}$ & $18 \mathrm{~m}$. & 1.188 & No & $\mathrm{Si}$ & $\mathrm{Si}$ & $\mathrm{NV}$ & $\mathrm{Si}$ & No & $\mathrm{Si}$ \\
\hline Marbury ${ }^{46} 1997$ & EEUU & $\mathrm{ECP}^{*}$ & 2 a. & 1.424 & No & $\mathrm{Si}$ & $\mathrm{Si}$ & $\mathrm{Si}$ & $\mathrm{Si}$ & $\mathrm{Si}$ & $\mathrm{Si}$ \\
\hline $\operatorname{Marx}^{88} 1995$ & EEUU & $\mathrm{ET}(\mathrm{CR})$ & & 2.090 & NA & No & $\mathrm{Si}$ & NA & No & No & $\mathrm{Si}$ \\
\hline McCormick $^{9} 1991$ & EEUU & $\mathrm{ECA}^{*}$ & $3 \mathrm{a}$. & 985 & $\mathrm{Si}$ & $\mathrm{Si}$ & $\mathrm{Si}$ & $\mathrm{Si}$ & $\mathrm{Si}$ & No & $\mathrm{Si}$ \\
\hline Nafstad ${ }^{50} 1999$ & Noruega & $\mathrm{ECP}$ & $4 \mathrm{a}$. & 4.863 & No & No & $\mathrm{Si}$ & No & No & $\mathrm{Si}$ & $\mathrm{Si}$ \\
\hline Nafstad $^{89} 2005$ & Noruega & $\mathrm{ECP}$ & $10 \mathrm{a}$. & 3.529 & No & No & $\mathrm{Si}$ & No & No & No & $\mathrm{Si}$ \\
\hline Nystad $^{58} 1999$ & Noruega & $\mathrm{ET}(\mathrm{CR})$ & & 1.447 & No & No & $\mathrm{Si}$ & $\mathrm{Si}$ & No & No & $\mathrm{Si}$ \\
\hline Ojembarrena $^{49} 1996$ & España & $\mathrm{ECP}^{*}$ & $10 \mathrm{~m}$. & 512 & No & $\mathrm{Si}$ & $\mathrm{Si}$ & NV & $\mathrm{Si}$ & $\mathrm{Si}$ & No \\
\hline Paradise $^{90} 1997$ & EEUU & $\mathrm{ECP} *$ & $2 \mathrm{a}$. & 3.663 & No & $\mathrm{Si}$ & $\mathrm{Si}$ & No & $\mathrm{Si}$ & $\mathrm{Si}$ & $\mathrm{Si}$ \\
\hline Pass $^{91} 1984$ & EEUU & $\mathrm{ECP}$ & $11 \mathrm{~m}$. & 165 & No & $\mathrm{Si}$ & No & NV & $\mathrm{Si}$ & No & No \\
\hline Petersson $^{92} 1990$ & Suecia & ECR & $3 \mathrm{a}$. & 489 & No & $\mathrm{Si}$ & $\mathrm{Si}$ & $\mathrm{Si}$ & $\mathrm{Si}$ & No & No \\
\hline Pettigrew $^{47} 2003$ & EEUU & $\mathrm{ECP}^{*}$ & $6 \mathrm{~m}$. & 674 & No & $\mathrm{Si}$ & $\mathrm{Si}$ & No & $\mathrm{Si}$ & $\mathrm{Si}$ & $\mathrm{Si}$ \\
\hline Presser $^{93} 1988$ & EEUU & $\mathrm{ET}(\mathrm{CR})$ & & 4.456 & NA & $\mathrm{Si}$ & $\mathrm{Si}$ & NA & $\mathrm{Si}$ & No & $\mathrm{Si}$ \\
\hline Rasmussen $^{94} 1990$ & Suecia & $\mathrm{ECP} *$ & $1 \mathrm{a}$. & 880 & No & $\mathrm{Si}$ & $\mathrm{Si}$ & $\mathrm{Si}$ & $\mathrm{Si}$ & $\mathrm{Si}$ & $\mathrm{Si}$ \\
\hline Rusconi $^{55} 1999$ & Italia & $\mathrm{ET}(\mathrm{CR})$ & & 18737 & No & No & $\mathrm{Si}$ & NA & $\mathrm{Si}$ & No & $\mathrm{Si}$ \\
\hline Rusconi $^{54,95} 2005$ & Italia & $\mathrm{ET}(\mathrm{CR})$ & & 20.016 & No & No & $\mathrm{Si}$ & NA & $\mathrm{Si}$ & No & $\mathrm{Si}$ \\
\hline Ruuska $^{96} 1991$ & Finlandia & $\mathrm{ECP}$ & $2 \mathrm{a}$. & 280 & No & $\mathrm{Si}$ & $\mathrm{Si}$ & $\mathrm{Si}$ & $\mathrm{Si}$ & No & No \\
\hline $\mathrm{Shi}^{97} 2000$ & EEUU & $\mathrm{ECP}$ & $2 \mathrm{~s}$. & 994 & NA & No & No & $\mathrm{Si}$ & $\mathrm{Si}$ & No & $\mathrm{Si}$ \\
\hline Silverstein $^{98} 2003$ & EEUU & ECR & $1 \mathrm{a}$. & 871 & No & $\mathrm{Si}$ & $\mathrm{Si}$ & NV & $\mathrm{Si}$ & No & $\mathrm{Si}$ \\
\hline Stahlberg ${ }^{99} 1980$ & Suecia & $\mathrm{ECP} *$ & $8 \mathrm{~s}$. & 69 & $\mathrm{Si}$ & $\mathrm{Si}$ & $\mathrm{Si}$ & $\mathrm{Si}$ & $\mathrm{Si}$ & $\mathrm{Si}$ & $\mathrm{Si}$ \\
\hline Strangert ${ }^{100,101} 1976$ & Suecia & $\mathrm{ECP}$ & $8 \mathrm{~m}$. & 165 & No & $\mathrm{Si}$ & $\mathrm{Si}$ & $\mathrm{Si}$ & $\mathrm{Si}$ & No & No \\
\hline Svanes $^{7} 2002$ & Multinacional & $\mathrm{ET}(\mathrm{CR})$ & & 18.530 & No & No & $\mathrm{Si}$ & NA & No & No & $\mathrm{Si}$ \\
\hline Tainio $^{102} 1988$ & Finlandia & $\mathrm{ECP} *$ & 2 a. & 183 & No & $\mathrm{Si}$ & $\mathrm{Si}$ & $\mathrm{Si}$ & $\mathrm{Si}$ & $\mathrm{Si}$ & $\mathrm{Si}$ \\
\hline Toroyan ${ }^{103} 2003$ & Gran Bretaña & $\mathrm{ECA}^{*}$ & $18 \mathrm{~m}$. & 143 & $\mathrm{Si}$ & $\mathrm{Si}$ & $\mathrm{Si}$ & $\mathrm{Si}$ & $\mathrm{Si}$ & No & No \\
\hline Wald $^{104} 1991$ & EEUU & $\mathrm{ECP}$ & 3 a. & 244 & No & $\mathrm{Si}$ & $\mathrm{Si}$ & $\mathrm{Si}$ & $\mathrm{Si}$ & No & No \\
\hline
\end{tabular}

Diseño: ECP estudio de cohortes prospectivo; ECR estudio de cohortes retrospectivo; ECA ensayo controlado aleatorizado; ET(CR) estudio transversal (cohorte retrospectiva); Tiempo de seguimiento: a. años; m. meses; s. semanas; No aplicable a ET(CR); Criterios de calidad: A) homogeneidad de los grupos; B) medición válida y homogénea de exposición (B1) y efecto (B2); C) seguimiento completo; D) control de la relación temporal (D1) y dosisrespuesta (D2); E) estratificación o análisis multivariante. NV no valorable; NA no aplicable.

* Trabajos seleccionados para el análisis de sensibilidad de estudios de mayor rigor metodológico. 
comparados con niños control, que se captaban en centros de salud, muestreos domiciliarios o telefónicos. El seguimiento se realizaba mediante revisiones o visitas periódicas, o mediante cuestionarios postales o telefónicos repetidos. En algún estudio se recopilaba información de las bases de datos de sistemas sanitarios.

En los estudios retrospectivos se emplearon habitualmente muestreos poblacionales, con entrevistas personales, por correo o telefónicas, utilizando en algún caso la recopilación de información histórica, para contrastar el nivel de exposición.

En la tabla 1 se detalla la calidad metodológica de los estudios incluidos en la revisión. Sólo cinco trabajos planificaron la selección de cohortes expuesta y no expuesta, emparejadas por otros factores de riesgo. Los porcentajes de estudios con una adecuada valoración de la exposición y efecto fueron respectivamente de un 69,2\% y $88,5 \%$. La medición de la exposición resultó muy heterogénea, tanto en la validez del método (cuestionario) como en la precisión de la información recogida (presencia/ausencia de guardería, edad de inicio, horas al día, días a la semana, duración, número de compañeros, etc). Este aspecto es uno los principales problemas al que debemos enfrentarnos a la hora de integrar la información de distintos estudios. Aunque la mayoría de los autores optan por un análisis dicotómico de la exposición (cualquier asistencia a guardería con respecto ninguna) algunos trabajos detallan variaciones en función del nivel de exposición (asistencia precoz, larga duración, presencia de muchos compañeros, etc).

En seis estudios $(11,5 \%)$ el seguimiento no fue completo, mientras que en nueve $(17,3 \%)$ este aspecto no pudo valorarse por falta de información y en otros 13 (25\%) por ser estudios retrospectivos. El seguimiento de la relación temporal entre exposición y efecto fue correcto en el $78,8 \%$, mientras que sólo en un 32,7\% se valoró la existencia de relación entre nivel de exposición (horas o días de asistencia a guardería, número de niños en contacto, etc.) y grado de efecto. El 80,8\% de los trabajos realizaron ajustes multivariantes y/o estratificación de los resultados para controlar la existencia de posibles factores de confusión.

En la tabla 2 se presenta una síntesis de las distintas medidas de efecto consideradas en los trabajos incluidos, con los estimadores resumen calculados a partir de los datos originales, siguiendo modelos de efectos aleatorios, en el caso de que hubiera más de un estudio que empleara la misma medida de efecto. Cuando sólo un trabajo ofrecía resultados cuantificables para una medida de efecto los estimadores corresponden a los resultados de dicho estudio. En los trabajos en que se presentaba la información separada para centros (guarderías con más de seis niños) y unidades domiciliarias de asistencia de día, sólo se consideraron las comparaciones entre los centros y el grupo control.

Puede observarse la gran variación existente en enfermedades valoradas y medidas de efecto. Muy pocos trabajos coinciden en la utilización de los mismos criterios. $\mathrm{La}$ asistencia a guardería, en comparación con la asistencia en los propios hogares o por personas del entorno familiar, mostró un mayor riesgo en los primeros años de vida de infecciones respiratorias en general, especialmente las de vías respiratorias altas (catarro común, rinitis, otitis media aguda o con derrame), algunas de vías respiratorias bajas (bronquitis, bronquiolitis, crup, neumonía, sibilantes asociados a infección respiratoria) y diarreas agudas. Los resultados de algún estudio sugieren que este mayor riesgo de infección respiratoria en los primeros años de vida podría reducir el riesgo de asma en los siguientes años de la infancia, sin embargo la información existente es muy heterogénea y no concluyen- 
Tabla 2

Comparaciones entre asistencia a guardería y grupo control (referencia)

\begin{tabular}{|c|c|c|c|c|c|c|}
\hline Comparaciones / Criterio evaluación citas & $\mathbf{N}$ & Casos & Estimador & \multicolumn{2}{|l|}{ Efecto $[\mathrm{IC} 95 \%]$} & AS [IC95\%] \\
\hline \multicolumn{7}{|l|}{ Infecciones respiratorias agudas } \\
\hline Infección respiratoria aguda (IRA) $66,71,72,79,93,104$ & 7 & 8344 & $\mathrm{RR}$ & $1,80[1,34 ; 2,43]$ & *\# & $2,23[1,62 ; 3,07]$ \\
\hline Riesgo (DI) ${ }^{68,72,74,92}$ & 4 & 780 & RDI & $1,86[1,25 ; 2,76]$ & *\# & $2,33[2,14 ; 2,54]$ \\
\hline $\mathrm{N}^{\circ}$ episodios/año ${ }^{66}$ & 1 & 1037 & $\mathrm{DM}$ & $0,72[0,40 ; 1,04]$ & * & $0,72[0,40 ; 1,04]$ \\
\hline Riesgo ajustado de consulta por IRA ${ }^{73}$ & 1 & 190 & RR & $1,43[1,19 ; 1,72]$ & $*$ & \\
\hline Riesgo Ajustado IRA frecuentes ${ }^{61,72}$ & 2 & 1525 & $\mathrm{RR}$ & $2,74[0,79 ; 9,53]$ & \# & $2,74[0,79 ; 9,53]$ \\
\hline \multicolumn{7}{|l|}{ Función pulmonar } \\
\hline Reducción volumen espiratorio forzado $1^{\mathrm{er}} \min . \%^{67}$ & 1 & 1111 & DM & $2,05[0,39 ; 3,71]$ & * & \\
\hline \multicolumn{7}{|l|}{ Otitis media aguda } \\
\hline Otitis Media Aguda ${ }^{48,50,68,71,100,101}$ & 6 & 5846 & $\mathrm{RR}$ & $1,46[1,25 ; 1,71]$ & & \\
\hline $\mathrm{N}^{\circ}$ episodios/año ${ }^{66,99}$ & 2 & 1083 & DMP & $0,35[0,17 ; 0,53]$ & * & $0,35[0,17 ; 0,53]$ \\
\hline Exceso episodios/año ${ }^{65}$ & 1 & 339 & DM & $1,13[0,39 ; 1,87]$ & * & $1,13[0,39 ; 1,87]$ \\
\hline Riesgo OMA frecuentes ${ }^{49,50,66,76}$ & 4 & 3036 & RR & $1,90[1,23 ; 2,92]$ & *\# & $1,64[0,80 ; 3,37]$ \\
\hline Riesgo (DI) ${ }^{94,101}$ & 2 & 1058 & RDI & $1,63[0,62 ; 4,27]$ & & $1,02[0,76 ; 1,37]$ \\
\hline Riesgo ajustado (DI) a los 1-7 años ${ }^{85}$ & 1 & 1453 & RDI & $1,71[1,53 ; 1,91]$ & * & \\
\hline Riesgo ajustado OMA en 1er año de vida ${ }^{80}$ & 1 & 4868 & RR & $1,35[1,24 ; 1,47]$ & * & $1,35[1,24 ; 1,47]$ \\
\hline Riesgo ajustado OMA frecuentes ${ }^{52,61,75,88}$ & 6 & 10958 & $\mathrm{RR}$ & $1,71[1,48 ; 1,97]$ & *\# & $\mathbf{1 , 5 8}[1,45 ; 1,71]$ \\
\hline \multicolumn{7}{|l|}{ Diarreas o gastroenteritis agudas } \\
\hline Gastroenteritis $^{60,66,96}$ & 3 & 5162 & $\mathrm{RR}$ & $3,22[1,87 ; 5,57]$ & $*$ & $3,37[1,79 ; 6,32]$ \\
\hline $\mathrm{N}^{\circ}$ episodios/año ${ }^{66}$ & 1 & 1037 & $\mathrm{DM}$ & $0,25[0,08 ; 0,42]$ & * & $0,25[0,08 ; 0,42]$ \\
\hline Riesgo (DI) $)^{64,77,86,94}$ & 4 & 3302 & RDI & $1,39[1,10 ; 1,74]$ & $* \#$ & $1,59[1,34 ; 1,88]$ \\
\hline Riesgo gastroenteritis 49,96 & 2 & 497 & RR & $2,25[1,64 ; 3,09]$ & $*$ & $2,38[1,62 ; 3,49]$ \\
\hline Exceso episodios/año ${ }^{65}$ & 1 & 339 & DM & $-0,15[-0,37 ; 0,07]$ & & $-0,15[-0,37 ; 0,07]$ \\
\hline Riesgo ajustado gastroenteritis ${ }^{63,77,86,87}$ & 4 & 3291 & RR & $1,36[1,16 ; 1,60]$ & *\# & $\mathbf{1 , 4 0}[1,09 ; 1,80]$ \\
\hline \multicolumn{7}{|l|}{ Infecciones respiratorias vías bajas } \\
\hline Exceso episodios/año ${ }^{65}$ & 1 & 339 & DM & $0,19[-0,03 ; 0,41]$ & & $0,19[-0,03 ; 0,41]$ \\
\hline Riesgo ajustado primeros $2-5$ años de vida ${ }^{46,52,78,81,82,87}$ & 6 & 6228 & RR & $2,10[1,54 ; 2,87]$ & *\# & $\mathbf{2 , 1 0}[1,54 ; 2,87]$ \\
\hline Riesgo $^{100,101}$ & 2 & 247 & RR & $2,97[1,16 ; 7,57]$ & $*$ & \\
\hline Riesgo (DI) 1er año ${ }^{74}$ & 1 & 92 & RDI & $1,38[0,88 ; 2,15]$ & & \\
\hline \multicolumn{7}{|l|}{ Sibilantes asociados a IRA } \\
\hline Riesgo ajustado (DI $)^{46}$ & 1 & 1371 & RDI & $2,30[1,78 ; 2,96]$ & * & $2,30[1,78 ; 2,96]$ \\
\hline 1er episodio en 2 meses $^{48}$ & 1 & 1624 & RR & $2,07[1,43 ; 2,99]$ & $*$ & \\
\hline Riesgo episodios frecuentes ${ }^{49}$ & 1 & 326 & RR & $4,59[1,97 ; 10,67]$ & * & $4,59[1,97 ; 10,67]$ \\
\hline Riesgo ajustado consulta sibilantes $1^{\text {os }} 6 \mathrm{~m}$. vida ${ }^{47}$ & 1 & 669 & $\mathrm{RR}$ & $2,30[1,51 ; 3,50]$ & $*$ & $2,30[1,51 ; 3,50]$ \\
\hline \multicolumn{7}{|l|}{ Bronquiolitis } \\
\hline Riesgo ajustado (DI) ${ }^{46}$ & 1 & 1371 & $\mathrm{RR}$ & $1,80[1,30 ; 2,49]$ & $*$ & $\mathbf{1 , 8 0}[1,30 ; 2,49]$ \\
\hline \multicolumn{7}{|l|}{ Neumonía } \\
\hline Riesgo ajustado (DI) ${ }^{46,85}$ & 2 & 2824 & RR & $1,73[1,31 ; 2,27]$ & $*$ & $\mathbf{1 , 7 0}[1,25 ; 2,31]$ \\
\hline Riesgo (DI) ${ }^{94}$ & 1 & 893 & RDI & $1,68[0,55 ; 5,14]$ & & $1,68[0,55 ; 5,14]$ \\
\hline \multicolumn{7}{|l|}{ Bronquitis } \\
\hline Riesgo ajustado (DI) ${ }^{46}$ & 1 & 1371 & RR & $2,10[1,59 ; 2,77]$ & * & $\mathbf{2 , 1 0}[1,59 ; 2,77]$ \\
\hline Riesgo ajustado consulta por bronquitis $1^{\text {os }} 6 \mathrm{~m}$. vida ${ }^{47}$ & 1 & 669 & RR & $1,71[0,71 ; 4,11]$ & & $1,71[0,71 ; 4,11]$ \\
\hline \multicolumn{7}{|l|}{ Crup } \\
\hline $\mathrm{N}^{\circ}$ episodios/año ${ }^{66}$ & 1 & 1037 & $\mathrm{DM}$ & $0,16[0,05 ; 0,27]$ & * & $0,16[0,05 ; 0,27]$ \\
\hline Riesgo ajustado (DI) ${ }^{46}$ & 1 & 1371 & $\mathrm{RR}$ & $1,70[1,28 ; 2,26]$ & * & $\mathbf{1 , 7 0}[1,28 ; 2,26]$ \\
\hline cruz frecuentes ${ }^{50,66}$ & 2 & 2597 & RR & $2,09[1,18 ; 3,71]$ & * & $3,03[1,33 ; 6,92]$ \\
\hline Riesgo ajustado cruz frecuentes ${ }^{50}$ & 1 & 1560 & $\mathrm{RR}$ & $1,72[0,95 ; 3,12]$ & & \\
\hline \multicolumn{7}{|l|}{ Catarro común } \\
\hline 1er episodio en 2 meses $^{48}$ & 1 & 1390 & $\mathrm{RR}$ & $1,76[1,41 ; 2,20]$ & * & \\
\hline Catarros frecuentes a los 2 años $^{56}$ & 1 & 447 & RR & $1,56[1,18 ; 2,07]$ & $*$ & $1,56[1,18 ; 2,07]$ \\
\hline Catarros frecuentes a los 6 años ${ }^{56}$ & 1 & 447 & RR & $0,59[0,28 ; 1,21]$ & & $0,59[0,28 ; 1,21]$ \\
\hline Catarros frecuentes a los 13 años $^{56}$ & 1 & 447 & RR & $1,04[0,42 ; 2,57]$ & & $1,04[0,42 ; 2,57]$ \\
\hline Riesgo ajustado Catarros frecuentes 1-2 años ${ }^{52,56}$ & 2 & 945 & RR & $1,24[1,03 ; 1,49]$ & * & $\mathbf{1 , 2 4}[1,03 ; 1,49]$ \\
\hline Riesgo ajustado (DI) 1-7años ${ }^{85}$ & 1 & 1453 & RDI & $1,22[0,59 ; 2,52]$ & & \\
\hline \multicolumn{7}{|l|}{ Rinitis } \\
\hline $\mathrm{N}^{\circ}$ episodios/mes ${ }^{99}$ & 1 & 46 & $\mathrm{DM}$ & $0,65[0,14 ; 1,16]$ & * & $0,65[0,14 ; 1,16]$ \\
\hline \multicolumn{7}{|l|}{ Otitis media con derrame } \\
\hline Riesgo OM con derrame ${ }^{90,94,103}$ & 3 & 2915 & $\mathrm{RR}$ & $1,60[1,29 ; 1,98]$ & * & $1,60[1,29 ; 1,98]$ \\
\hline Riesgo ajustado OM con derrame $\mathrm{O}^{62,69,70}$ & 2 & 1832 & RR & $1,60[0,87 ; 2,95]$ & & $\mathbf{2 , 4 3}[1,21 ; 4,88]$ \\
\hline
\end{tabular}


Tabla 2 (continuación)

Comparaciones entre asistencia a guardería y grupo control (referencia)

\begin{tabular}{|c|c|c|c|c|c|}
\hline Comparaciones / Criterio evaluación citas & $\mathbf{N}$ & Casos & Estimador & Efecto $[\mathrm{IC} 95 \%]$ & AS $[\mathrm{IC} 95 \%]$ \\
\hline \multicolumn{6}{|l|}{ Asma } \\
\hline Asma a los 6-13 años $55,57,83$ & 3 & 19563 & RR & $0,66[0,40 ; 1,11]$ & $0,46[0,21 ; 1,00]$ \\
\hline Riesgo (DI) $1-5$ años ${ }^{94}$ & 1 & 893 & RR & $5,47[2,05 ; 14,57] *$ & $5,47[2,05 ; 14,57]$ \\
\hline Riesgo ajustado sibilantes recurrentes $1-2^{\circ}$ año ${ }^{46,51,53}$ & 2 & 1766 & RR & $1,35[0,88 ; 2,07]$ & $1,35[0,88 ; 2,07]$ \\
\hline Riesgo ajustado sibilantes recurrentes 6-7 años ${ }^{51,53}$ & 1 & 498 & RR & $0,53[0,25 ; 1,13]$ & $0,53[0,25 ; 1,13]$ \\
\hline Riesgo ajustado asma a los 3-5 años ${ }^{50,51,53}$ & 2 & 2058 & $\mathrm{RR}$ & $1,23[0,70 ; 2,18]$ & $0,82[0,37 ; 1,79]$ \\
\hline Riesgo ajustado asma a los 6-13 años ${ }^{53,57,58,89}$ & 4 & 5481 & RR & $0,86[0,59 ; 1,26]$ & $0,56[0,33 ; 0,95]$ \\
\hline Riesgo ajustado asma en edad adulta ${ }^{7}$ & 1 & 18530 & RR & $1,01[0,94 ; 1,07]$ & \\
\hline Riesgo ajustado sibilantes precoces transitorios ${ }^{54,55}$ & 2 & 23263 & RR & $1,44[1,16 ; 1,79] * \#$ & \\
\hline Riesgo ajustado sibilantes precoces persistentes 6-7 años ${ }^{54,55}$ & 2 & 33263 & RR & $0,93[0,82 ; 1,07]$ & \\
\hline Riesgo ajustado sibilantes inicio tardío 6-7 años ${ }^{54,55}$ & 2 & 33263 & $\mathrm{RR}$ & $0,83[0,67 ; 1,02]$ & \\
\hline \multicolumn{6}{|l|}{ Uso recursos asistenciales } \\
\hline Ingreso año previo ${ }^{65}$ & 1 & 531 & RR & $5,10[1,42 ; 18,30] *$ & $5,10[1,42 ; 18,30]$ \\
\hline Media de ingresos en los primeros 3 años ${ }^{9}$ & 1 & 857 & $\mathrm{DM}$ & $0,05[-0,13 ; 0,23]$ & $0,05[-0,13 ; 0,23]$ \\
\hline Media de consultas en los primeros 3 años ${ }^{9}$ & 1 & 857 & $\mathrm{DM}$ & $1,60[-0,41 ; 3,61]$ & $1,60[-0,41 ; 3,61]$ \\
\hline Riesgo ajustado de ingreso ${ }^{98}$ & 1 & 618 & RR & $1,82[0,81 ; 4,10]$ & \\
\hline Riesgo ajustado de consulta en urgencias ${ }^{98}$ & 1 & 618 & RR & $1,74[1,13 ; 2,69] *$ & \\
\hline \multicolumn{6}{|l|}{ Infecciones respiratorias vías altas } \\
\hline Riesgo ajustado al 1-2 años de vida ${ }^{81,82}$ & 2 & 2219 & RR & $1,88[1,67 ; 2,11] *$ & $\mathbf{1 , 8 8}[1,67 ; 2,11]$ \\
\hline \multicolumn{6}{|l|}{ Portador de neumococo } \\
\hline Riesgo ajustado de 2 a 24 meses $^{84}$ & 1 & 100 & OR & $1,30[0,77 ; 2,20]$ & $1,30[0,77 ; 2,20]$ \\
\hline \multicolumn{6}{|l|}{ Infección por citomegalovirus } \\
\hline Prevalencia $^{91}$ & 1 & 160 & RR & $3,27[1,82 ; 5,87] *$ & \\
\hline \multicolumn{6}{|l|}{ Síntomas respiratorios } \\
\hline Riesgo ajustado síntomas moderados en 2 semanas $^{97}$ & 1 & 994 & OR & $1,32[1,11 ; 1,56] *$ & \\
\hline Riesgo ajustado síntomas graves en 2 semanas ${ }^{97}$ & 1 & 994 & OR & $1,18[0,85 ; 1,64]$ & \\
\hline
\end{tabular}

N: número de estudios incluídos; DI: densidad de incidencia; Todas las medidas han sido calculadas a partir de los datos publicados utilizando modelos de efectos aleatorios: DM: diferencia de medias; DMP: diferencia de medias ponderada; RR: riesgo relativo; OR odds ratio; RDI: razón de densidades de incidencia; IC95\%: intervalo de confianza del 95\%; Con asterisco indica efecto significativo ( $<<0,05)$; \# heterogeneidad entre estudios estadísticamente significativa (sólo calculable si hay más de 1 estudio). AS: análisis de sensibilidad con estudios de mayor rigor metodológico (en blanco si no hay estudios). Se destacan en negrita los estimadores de efecto ajustados de mayor relevancia. No hay heterogeneidad.

te. Las diferencias en cuanto a la utilización de recursos asistenciales son muy escasas, mientras que la información disponible con respecto a otras infecciones está sustentada en pocos estudios y de pequeño tamaño (infección por citomegalovirus o neumococo).

Se realizaron diagramas de embudo ("funnel plot") de las comparaciones que contaban con más de cinco trabajos, no encontrando imágenes que sugirieran la posible existencia de sesgos de publicación.

Aunque en el análisis global encontramos heterogeneidad significativa para varias medidas de riesgo (tabla 2) cuando seleccionamos sólo los trabajos con mayor rigor metodológico (tabla 1) y que ofrecían estimadores de efecto ajustados (mediante análisis multivariante) con control de posibles factores de confusión, la heterogeneidad desaparecía (análisis de sensibilidad). En la tabla 2 se destacan en negrita los estimadores de riesgo considerados más relevantes, por valorar medidas ajustadas obtenidas habitualmente de más de un estudio con aceptable rigor metodológico. Considerando sólo estos resultados podemos resumir que para la asistencia a guardería el riesgo de infección respiratoria alta se incrementa un $88 \%$, para la otitis media un $58 \%$, para la otitis media con derrame un $143 \%$, para las infecciones respiratorias bajas un $110 \%$ (neumonías 70\%; bronquiolitis $80 \%$; bronquitis $110 \%$ ) y para las 
diarreas/gastroenteritis agudas un $40 \%$. Transformando los riesgos estimados en proporciones atribuibles la asistencia a guardería podría ser la responsable de entre un 33 y un $50 \%$ de los episodios de infecciones respiratorias y gastroenteritis en la población expuesta.

\section{COMENTARIOS}

La asistencia a guardería durante los primeros años de vida parece entrañar un incremento del riesgo de adquirir diversas infecciones. Este aumento de riesgo parece consistente a lo largo del tiempo y entre diferentes entornos sociales y geográficos. $\mathrm{La}$ evidencia disponible parece apoyar suficientemente la existencia de este riesgo para infecciones respiratorias agudas, otitis en particular, y gastroenteritis. La información disponible para algunas infecciones respiratorias de vías bajas es escasa, aunque también apunta en la misma dirección.

La valoración del riesgo de asma en relación con la asistencia en guardería resulta problemática. Por un lado, deberíamos diferenciar formas transitorias de espasticidad bronquial, asociadas a infecciones respiratorias en los primeros años de vida con formas persistentes en años posteriores. Distintos estudios muestran que la asistencia a guardería incrementa el riesgo de tener sibilantes hasta los 3-4 años ${ }^{46-53}$, probablemente en relación con formas transitorias ${ }^{54,55}$, pasando a mostrar posteriormente un efecto protector entre los 6 y 13 años ${ }^{51-53,56-58,}$ efecto que progresivamente se reduce hasta atenuarse en la edad adulta ${ }^{7}$. Es posible que la ausencia de significación estadística de los estudios que analizan el riesgo en edades ya más avanzadas pueda deberse a dificultades para identificar con precisión el nivel de exposición en los estudios retrospectivos realizados en esas épocas.

Aunque la dirección de los efectos observados parece bastante estable entre es- tudios, existen diferencias en su magnitud. Estas discordancias podrían reflejar verdaderas diferencias entre distintos sistemas sanitarios o socioculturales, pero también podrían deberse a variaciones en los criterios de medición de la exposición y sus efectos. De hecho, uno de los principales problemas a los que nos enfrentamos en esta revisión es la heterogeneidad en la medición de la exposición. Este hecho no parece que pueda influir en la dirección del efecto pero sí en la estimación de su magnitud. Cualquier estimador que utilicemos va a originar medidas más o menos extremas en función del grado de exposición que escojamos para evaluar el efecto de las guarderías. No es lo mismo que consideremos como grupo expuesto cualquier contacto en el tiempo con la guardería que grupos seleccionados con asistencia precoz, estancias prolongadas o en centros con numerosos compañeros. En este mismo sentido resulta comprometido englobar como una misma categoría guarderías de diferente calidad, en cuanto a estructura, personal o procedimientos higiénicos.

También hemos observado una gran heterogeneidad en las medidas de efecto elegidas en los distintos estudios. Es escasa la utilización de medidas de mayor relevancia clínica, como la existencia de procesos recurrentes (por ejemplo más de cinco otitis/año), empleándose con más frecuencia otras medidas cualitativas (al menos un episodio) o cuantitativas (número de episodios). Esta dispersión en la elección de medidas de efecto se traduce en que no haya trabajos suficientes para un análisis agrupado en algunas de ellas. Por otra parte, si tenemos en cuenta la heterogeneidad basal de la mayoría de las cohortes estudiadas, la validez de estimadores que no contemplen un ajuste multivariante por otros factores de riesgo parece cuestionable. De hecho, el análisis global para algunas medidas de efecto mostraba heterogeneidad significativa entre estudios. Sin embargo, cuando seleccionamos los estudios con mayor ca- 
lidad metodológica, la heterogeneidad estadística desaparecía, por lo que sus estimadores de riesgo resumen pueden considerarse fiables.

En esta revisión hemos excluido las comparaciones con las unidades de atención de día en domicilios ("family daycare"), práctica muy extendida en diversos países, donde pueden estar supervisadas y financiadas por instituciones públicas, pero que no se adapta a nuestra realidad. Los estudios que analizan por separado este grupo encuentran riesgos intermedios entre los observados en las guarderías más grandes ("day care center") y el grupo de atención en su domicilio o por personal del entorno familiar ${ }^{12}$. Esta revisión debe ser sin duda ampliada con un análisis separado de esta categoría, o utilizando métodos estadísticos que evalúen tendencias o controlen el nivel de exposición.

Nuestra búsqueda bibliográfica incluyó las bases de datos de PubMed, Colaboración Cochrane e Índice Médico Español, así como la revisión de citas bibliográficas de todos los artículos seleccionados. Consideramos que el examen de citas permitió aumentar suficientemente la sensibilidad de la búsqueda, ya que una búsqueda realizada a posteriori en EMBASE solo localizó un estudio suplementario. Al igual que en otras revisiones sistemáticas no podemos descartar que pueda existir cierto sesgo de publicación que esconda una tendencia a no publicar trabajos con resultados negativos o a derivarlos a publicaciones menos accesibles, a pesar de que el análisis gráfico (diagramas de embudo) no lo sugiere.

Las principales causas de exclusión de estudios en esta revisión fueron el diseño de casos y controles y los estudios de cohorte única (guardería) sin grupo control. Los estudios de casos y controles fueron descartados a priori por protocolo, al considerar que eran más propensos a producir estimaciones sesgadas de efecto. Este hecho ha sido ad- vertido en una revisión sistemática que analizaba el efecto de la guardería en la incidencia de diabetes mellitus tipo $\mathrm{I}^{5}$. Resulta igualmente ilustrativo que un estudio de casos y controles excluido ${ }^{17}$, en el que se analizaba el riesgo de asma a los 3-4 años de edad, muestre un fuerte efecto protector de la guardería no observado en otros estudios de cohortes. El diseño de cohortes se adapta mejor a la valoración de la exposición y el efecto en los primeros años de vida, aunque los estudios de casos y controles permiten explorar el papel de factores de riesgo en enfermedades más raras. En este sentido, diversos estudios de casos y controles han identificado en los niños atendidos en guarderías un mayor riesgo de infección neumocócica invasiva ${ }^{18}$ y de enfermedad de Crohn ${ }^{16}$, y un menor riesgo de diabetes mellitus tipo $\mathrm{I}^{5}$, no encontrando diferencias en relación al neuroblastoma ${ }^{19}$ o la leucemia linfoblástica aguda ${ }^{6}$.

Implicaciones para la práctica: Conocer que la asistencia de día en guardería incrementa el riesgo de determinadas infecciones tiene interés para los médicos de atención primaria que tratan a estos niños, para los especialistas que controlan enfermos crónicos, cuya enfermedad puede verse agravada por dicho riesgo, para los adultos que cuidan a estos niños y pueden verse expuestos a dichas infecciones y para las autoridades sanitarias que son responsables de la prevención, vigilancia y manejo de brotes epidémicos que puedan aparecer en esas instituciones. Los estimadores de riesgo asociados a la asistencia en guarderías muestran valores más discretos que los observados en otros modelos de enfermedad, pero si tenemos en cuenta la gran prevalencia de la exposición estudiada y la alta frecuencia de las infecciones aquí analizadas, puede comprenderse la repercusión del problema.

A pesar del riesgo observado las circunstancias sociales actuales limitan cualquier propuesta que trate de disminuir la 
asistencia en guarderías de nuestros niños más pequeños. Podrían proponerse alternativas para reducir el nivel de exposición, disminuyendo las horas de asistencia o el número de niños por centro, educando a los cuidadores sobre los principios de la transmisión de enfermedades, extremando las medidas de higiene y dictando normas claras para el manejo de los casos aparecidos y la protección de sus contactos ${ }^{4,59}$. No obstante, es posible que, salvo en niños con enfermedades crónicas o inmunodeficiencias, el riesgo sea aceptable, pudiendo en algunos casos verse compensado por el potencial beneficio de socialización y estimulación que la guardería lleva implícito ${ }^{8}$.

\section{Implicaciones para la investigación:}

Parece necesaria la realización de nuevos estudios válidos que analicen la magnitud del riesgo de la asistencia a guarderías, preferiblemente con diseños prospectivos, en los que la medición de la exposición sea lo más precisa posible, empleando diversos parámetros de graduación (edad de inicio, horas, días, número de compañeros, etc.) y puntos de corte alternativos. Asimismo, la valoración de la ocurrencia de enfermedad debe realizarse de forma equiparable en los grupos expuestos y no expuestos, empleando métodos de recogida complementarios (diarios o cuestionarios periódicos a cuidadores y padres, y revisión de registros médicos) que traten de mejorar su validez y precisión, con resultados tanto cuantitativos (incidencia, densidad de incidencia) como cualitativos (episodios frecuentes) y ajustes multivariantes. Por otra parte, debe también proponerse la realización de estudios de evaluación de la efectividad de medidas de intervención encaminadas a reducir la incidencia de enfermedad en este grupo de riesgo.

\section{CONCLUSIONES}

La asistencia a guardería se asocia a un significativo aumento del riesgo de enfer- medad infecciosa aguda en la infancia. Este riesgo es consistente a lo largo del tiempo y entre diferentes entornos sociales y geográficos. Considerando los trabajos con mayor rigor metodológico y con estimadores de efecto ajustados, la asistencia a guardería se asocia a un incremento de riesgo de infección respiratoria alta, otitis media aguda, otitis media con derrame, infecciones respiratorias bajas y gastroenteritis agudas. La asistencia a guardería podría ser la responsable de entre un $33 \%$ y un $50 \%$ de los episodios de infección respiratoria y gastroenteritis en la población expuesta. No obstante, a la hora de decidir la conveniencia de que un niño sea atendido o no en una guardería debe considerarse si la familia dispone de sistemas de cuidado alternativos, así como los posibles beneficios de socialización y estimulación de la escolarización precoz. Esta información tiene importantes implicaciones para la investigación, la práctica clínica, las autoridades sanitarias y la sociedad.

\section{BIBLIOGRAFÍA}

1. Yeandle S, Gore T, Herrington A. Employment, family and community activities. A new balance for women and men. Luxembourg: Office for Official Publication of the European Communities; 1999.

2. Instituto Nacional de Estadística. Fuente: Ministerio de Educación y Ciencia. Alumnado. Régimen general. Curso 2003-2004. [fecha de consulta: 10-X-2006]. Disponible en: http: // www.ine.es/inebase/cgi/axi?AXIS_PATH=/inebase/temas/t13/p001/e03/a2003-2004/10/\&FILE_AXIS=amg10002.px\&CGI_DEFAULT=/in ebase/temas/cgi.opt\&COMANDO=SELECCION\&CGI_URL=/inebase/cgi/.

3. Encuesta de Población Activa. Módulo especial 2005: Módulo de conciliación entre la vida laboral y la familiar. [Fecha de consulta 10-X2006]. Disponible en: http: //www.ine.es/inebase/cgi/axi?AXIS_PATH=/inebase/temas/t22/e3 08/meto_05/modulo/2005/10/\&FILE_AXIS=01 001.px\&CGI_DEFAULT=/inebase/temas/cgi.o pt\&COMANDO=SELECCION\&CGI_URL $=/ \mathrm{i}$ nebase/cgi/. 
4. Barros FC. Child-care attendance and common morbidity: evidence of association in the literature and questions of design. Rev Saude Publica. $1999 ; 33$ : 98-106.

5. Kaila B, Taback SP. The effect of day care exposure on the risk of developing type 1 diabetes: a meta-analysis of case-control studies. Diabetes Care. 2001; 24: 1353-8.

6. Rosenbaum PF, Buck GM, Brecher ML. Early child-care and preschool experiences and the risk of childhood acute lymphoblastic leukemia. Am J Epidemiol. 2000; 152: 1136-44.

7. Svanes C, Jarvis D, Chinn S, Omenaas E, Gulsvik A, Burney P. Early exposure to children in family and day care as related to adult asthma and hay fever: results from the European Community Respiratory Health Survey. Thorax. 2002; 57: 945-50.

8. Zoritch B, Roberts I, Oakley A. The health and welfare effects of day-care: a systematic review of randomised controlled trials. Soc Sci Med. 1998; 47: 317-27.

9. McCormick MC, Brooks-Gunn J, Shapiro S, Benasich AA, Black G, Gross RT. Health care use among young children in day care. Results in a randomized trial of early intervention. JAMA. 1991; 265: 2212-7.

10. Chalmers I, Altman DG. Systematic Reviews. London: BMJ Publishing Group; 1995.

11. Schulz KF, Chalmers I, Hayes RJ, Altman DG. Empirical evidence of bias. JAMA. 1995; 273 : 408-12.

12. Uhari M, Mantysaari K, Niemela M. A metaanalytic review of the risk factors for acute otitis media. Clin Infect Dis. 1996; 22: 1079-83.

13. Rovers MM, Zielhuis GA, Ingels $\mathrm{K}$, van der Wilt GJ. Day-care and otitis media in young children: a critical overview. Eur J Pediatr. 1999; 158: 1-6.

14. Fernández E, García AM. Estudios epidemiológicos (STROBE). Med Clin (Barc). 2005; 125 Supl 1: 43-8.

15. Zhang J, Yu KF. What's the Relative Risk?: A Method of Correcting the Odds Ratio in Cohort Studies of Common Outcomes. JAMA. 1998; 280: 1690-1.

16. Amre DK, Lambrette P, Law L, Krupoves A, Chotard V, Costea F, et al. Investigating the hygiene hypothesis as a risk factor in pediatric onset Crohn's disease: a case-control study. Am J Gastroenterol. 2006; 101: 1005-11.

17. Infante-Rivard C, Amre D, Gautrin D, Malo JL. Family size, day-care attendance, and breastfeeding in relation to the incidence of childhood asthma. Am J Epidemiol. 2001; 153: 653-8.

18. Levine OS, Farley M, Harrison LH, Lefkowitz L, McGeer A, Schwartz B. Risk factors for invasive pneumococcal disease in children: a population-based case-control study in North America. Pediatrics. 1999; 103: E28.

19. Menegaux F, Olshan AF, Neglia JP, Pollock BH, Bondy ML. Day care, childhood infections, and risk of neuroblastoma. Am J Epidemiol. 2004; 159: $843-51$.

20. Vinther B, Elbrond O, Pedersen CB. Otitis media in childhood. Socio-medical aspects vith special reference to day-care and housing conditions. Acta Otolaryngol Suppl. 1982; 386: 121-3.

21. Andreev A, Arjas E. Acute middle ear infection in small children: a Bayesian analysis using multiple time scales. Lifetime Data Anal. 1998; 4: 121-37.

22. Oja H, Alho OP, Laara E. Model-based estimation of the excess fraction (attributable fraction): day care and middle ear infection. Stat Med. 1996; 15: 1519-34.

23. Carabin H, Gyorkos TW, Soto JC, Penrod J, Joseph L, Collet JP. Estimation of direct and indirect costs because of common infections in toddlers attending day care centers. Pediatrics. 1999; 103: 556-64.

24. Gomez-Barreto D, Calderon-Jaimes E, Rodriguez RS, Espinosa LE, Vina-Flores L, JimenezRojas V. Carriage of antibiotic-resistant pneumococci in a cohort of a daycare center. Salud Publica Mex. 2002; 44: 26-32.

25. Costa Bou X. ¿Tan malo es el primer año en la guardería? An Esp Pediatr. 1989; 31: 449-54.

26. Cordell RL, Waterman SH, Chang A, Saruwatari M, Brown M, Solomon SL. Provider-reported illness and absence due to illness among children attending child-care homes and centers in San Diego, Calif. Arch Pediatr Adolesc Med. 1999; 153: 275-80.

27. Leeder SR, Corkhill RT, Irwig LM, Holland WW, Colley JRT. Influence of family factors on asthma and wheezing during the first five years of life. Br J Prev Soc Med. 1976; 30: 213-8. 
28. Melander E, Molstad S, Persson K, Hansson HB, Soderstrom M, Ekdahl K. Previous antibiotic consumption and other risk factors for carriage of penicillin-resistant Streptococcus pneumoniae in children. Eur J Clin Microbiol Infect Dis. 1998; 17: 834-8.

29. Nandi-Lozano E, Espinosa LE, Vinas-Flores L, Avila-Figueroa C. Infección respiratoria aguda en niños que acuden a un centro de desarrollo infantil. Salud Publica Mex. 2002; 44: 201-6.

30. Rosenfeldt V, Vesikari T, Pang XL, Zeng SQ, Tvede M, Paerregaard A. Viral etiology and incidence of acute gastroenteritis in young children attending day-care centers. Pediatr Infect Dis J. 2005; 24: 962-5.

31. Schwartz B, Giebink GS, Henderson FW, Reichler MR, Jereb J, Collet JP. Respiratory infections in day care. Pediatrics. 1994; 94: 1018-20.

32. Yalcin SS, Turul B, Cetinkaya S, Cakir B, Yilmaz A. Effect of total attending period on infection episode rate in a child-care center. Pediatr Int. 2004; 46: 555-60.

33. Dales RE, Cakmak S, Brand K, Judek S. Respiratory illness in children attending daycare. Pediatr Pulmonol. 2004; 38: 64-9.

34. Anme T, Segal UA. Implications for the development of children in over 11 hours of centrebased care. Child Care Health Dev. 2004; 30: 345-52.

35. Skull SA, Ford-Jones EL, Kulin NA, Einarson TR, Wang EE. Child care center staff contribute to physician visits and pressure for antibiotic prescription. Arch Pediatr Adolesc Med. 2000; 154: 180-3.

36. Thrane N, Olesen C, Md JT, Sondergaard C, Schonheyder HC, Sorensen HT. Influence of day care attendance on the use of systemic antibiotics in 0- to 2-year-old children. Pediatrics. 2001; 107: E76.

37. Dunais B, Pradier C, Carsenti H, Sabah M, Mancini G, Fontas E, et al. Influence of child care on nasopharyngeal carriage of Streptococcus pneumoniae and Haemophilus influenzae. Pediatr Infect Dis J. 2003; 22: 589-92.

38. Johansen AS, Leibowitz A, Waite LJ. Child care and children's illness. Am J Public Health. 1988; 78: 1175-7.

39. Sorri M, Alho OP, Oja H. Dynamic multivariate modelling: day care and consultation rate for acute otitis media. Acta Otolaryngol. 1996; 116: 299-301.

40. Aniansson G, Alm B, Andersson B, Hakansson A, Larsson P, Nylen O, et al. A prospective cohort study on breast-feeding and otitis media in Swedish infants. Pediatr Infect Dis J. 1994; 13: 183-8.

41. Super CM, Keefer CH, Harkness S. Child care and infectious respiratory disease during the first two years of life in a rural Kenyan community. Soc Sci Med. 1994; 38: 227-9.

42. Sznajder M, Stheneur C, Albonico V, Dib S, Cau D, Chevallier B. Respiratory development of 5- to 6-year-old children experiencing a first bronchiolitis episode before age one. Allerg Immunol (Paris). 2005; 37: 392-6.

43. Dewey C, Midgeley E, Maw R. The relationship between otitis media with effusion and contact with other children in a british cohort studied from 8 months to $31 / 2$ years. The ALSPAC Study Team. Avon Longitudinal Study of Pregnancy and Childhood. Int J Pediatr Otorhinolaryngol. 2000; 55: 33-45.

44. Sipila M, Karma P, Pukander J, Timonen M, Kataja M. The Bayesian approach to the evaluation of risk factors in acute and recurrent acute otitis media. Acta Otolaryngol. 1988; 106: 94-101.

45. Vernacchio L, Lesko SM, Vezina RM, Corwin MJ, Hunt CE, Hoffman HJ, et al. Racial/ethnic disparities in the diagnosis of otitis media in infancy. Int J Pediatr Otorhinolaryngol. 2004; 68: 795-804.

46. Marbury MC, Maldonado G, Waller L. Lower respiratory illness, recurrent wheezing, and day care attendance. Am J Respir Crit Care Med. 1997; 155: 156-61.

47. Pettigrew MM, Khodaee M, Gillespie B, Schwartz K, Bobo JK, Foxman B. Duration of breastfeeding, daycare, and physician visits among infants 6 months and younger. Ann Epidemiol. 2003; 13: 431-5.

48. Collet JP, Ducruet T, Floret D, Cogan-Collet J, Honneger D, Boissel JP. Daycare attendance and risk of first infectious disease. Eur J Pediatr. 1991; 150: 214-6.

49. Ojembarrena Martínez E, Fernández de Pinedo Montoya R, Lafuente Mesanza P, Corera Sánchez M. Papel de la guardería y de la escolarización precoz en la incidencia de enfermedades infecciosas. An Esp Pediatr. 1996; 45: 45-8. 
50. Nafstad P, Hagen JA, Oie L, Magnus P, Jaakkola JJ. Day care centers and respiratory health. Pediatrics. 1999; 103: 753-8.

51. Celedon JC, Litonjua AA, Ryan L, Weiss ST, Gold DR. Day care attendance, respiratory tract illnesses, wheezing, asthma, and total serum IgE level in early childhood. Arch Pediatr Adolesc Med. 2002; 156: 241-5.

52. Celedon JC, Litonjua AA, Weiss ST, Gold DR. Day care attendance in the first year of life and illnesses of the upper and lower respiratory tract in children with a familial history of atopy. Pediatrics. 1999; 104: 495-500.

53. Celedon JC, Wright RJ, Litonjua AA, Sredl D, Ryan L, Weiss ST, et al. Day care attendance in early life, maternal history of asthma, and asthma at the age of 6 years. Am J Respir Crit Care Med. 2003; 167: 1239-43.

54. Rusconi F, Galassi C, Bellasio M, Piffer S, Lombardi E, Bonci E, et al. Fattori di rischio pre e perinatali e del primo anno di vita e sviluppo di wheezing in eta prescolare. Epidemiol Prev. 2005; 29 : 47-51.

55. Rusconi F, Galassi C, Corbo GM, Forastiere F, Biggeri A, Ciccone G, et al. Risk factors for early, persistent, and late-onset wheezing in young children. SIDRIA Collaborative Group. Am J Respir Crit Care Med. 1999; 160: 1617-22.

56. Ball TM, Holberg CJ, Aldous MB, Martinez FD, Wright AL. Influence of attendance at day care on the common cold from birth through 13 years of age. Arch Pediatr Adolesc Med. 2002; 156: 121-6.

57. Ball TM, Castro-Rodriguez JA, Griffith KA, Holberg CJ, Martinez FD, Wright AL. Siblings, day-care attendance, and the risk of asthma and wheezing during childhood. N Engl J Med. 2000; 343: 538-43.

58. Nystad W, Skrondal A, Magnus P. Day care attendance, recurrent respiratory tract infections and asthma. Int J Epidemiol. 1999; 28: 882-7.

59. Goodman RA, Osterholm MT, Granoff DM, Pickering LK. Infectious diseases and child day care. Pediatrics. 1984; 74: 134-9.

60. Alexander CS, Zinzeleta EM, Mackenzie EJ, Vernon A, Markowitz RK. Acute gastrointestinal illness and child care arrangements. Am J Epidemiol. 1990; 131: 124-31.

61. Alho OP, Koivu M, Sorri M, Rantakallio P. Risk factors for recurrent acute otitis media and res- piratory infection in infancy. Int J Pediatr Otorhinolaryngol. 1990; 19: 151-61.

62. Alho OP, Oja H, Koivu M, Sorri M. Risk factors for chronic otitis media with effusion in infancy. Each acute otitis media episode induces a high but transient risk. Arch Otolaryngol Head Neck Surg. 1995; 121: 839-43.

63. Barros H, Lunet N. Association between childcare and acute diarrhea: a study in Portuguese children. Rev Saude Publica. 2003; 37: 603-8.

64. Bartlett AV, Moore M, Gary GW, Starko KM, Erben JJ, Meredith BA. Diarrheal illness among infants and toddlers in day care centers. II. Comparison with day care homes and households. J Pediatr. 1985; 107: 503-9.

65. Bell DM, Gleiber DW, Mercer AA, Phifer R, Guinter RH, Cohen AJ, et al. Illness associated with child day care: a study of incidence and cost. Am J Public Health. 1989; 79: 479-84.

66. Collet JP, Burtin P, Gillet J, Bossard N, Ducruet T, Durr F. Risk of infectious diseases in children attending different types of day-care setting. Epicreche Research Group. Respiration. 1994; 61 Suppl 1: 16-9.

67. Demissie K, Ernst P, Joseph L, Becklake MR. The role of domestic factors and day-care attendance on lung function of primary school children. Respir Med. 1998; 92: 928-35.

68. Doyle AB. Incidence of illness in early group and family day-care. Pediatrics. 1976; 58: 607-13.

69. Engel J, Anteunis L, Volovics A, Hendriks J, Marres E. Prevalence rates of otitis media with effusion from 0 to 2 years of age: healthy-born versus high-risk-born infants. Int J Pediatr Otorhinolaryngol. 1999; 47: 243-51.

70. Engel J, Anteunis L, Volovics A, Hendriks J, Marres E. Risk factors of otitis media with effusion during infancy. Int J Pediatr Otorhinolaryngol. 1999; 48: 239-49.

71. Fleming DW, Cochi SL, Hightower AW, Broome CV. Childhood upper respiratory tract infections: to what degree is incidence affected by day-care attendance? Pediatrics. 1987; 79: 55-60.

72. Flores Hernandez S, Reyes Morales H, Perez Cuevas R, Guiscafre Gallardo H. The day care center as a risk factor for acute respiratory infections. Arch Med Res. 1999; 30: 216-23.

73. Forssell G, Hakansson A, Mansson NO. Risk factors for respiratory tract infections in chil- 
dren aged 2-5 years. Scand J Prim Health Care. 2001; 19: 122-5.

74. Gardner G, Frank AL, Taber LH. Effects of social and family factors on viral respiratory infection and illness in the first year of life. J Epidemiol Community Health. 1984; 38: 42-8.

75. Hardy AM, Fowler MG. Child care arrangements and repeated ear infections in young children. Am J Public Health. 1993; 83: 1321-5.

76. Harsten G, Prellner K, Heldrup J, Kalm O, Kornfalt R. Recurrent acute otitis media. A prospective study of children during the first three years of life. Acta Otolaryngol. 1989; 107: 111-9.

77. Hillis SD, Miranda CM, McCann M, Bender D, Weigle K. Day care center attendance and diarrheal morbidity in Colombia. Pediatrics. 1992; 90: 582-8.

78. Holberg CJ, Wright AL, Martinez FD, Morgan WJ, Taussig LM. Child day care, smoking by caregivers, and lower respiratory tract illness in the first 3 years of life. Group Health Medical Associates. Pediatrics. 1993; 91: 885-92.

79. Hurwitz ES, Gunn WJ, Pinsky PF, Schonberger LB. Risk of respiratory illness associated with day-care attendance: a nationwide study. Pediatrics. 1991; 87: 62-9.

80. Kero P, Piekkala P. Factors affecting the occurrence of acute otitis media during the first year of life. Acta Paediatr Scand. 1987; 76: 618-23.

81. Koch A, Molbak K, Homoe P, Sorensen P, Hjuler $\mathrm{T}$, Olesen ME, et al. Risk factors for acute respiratory tract infections in young Greenlandic children. Am J Epidemiol. 2003; 158: 374-84.

82. Koopman LP, Smit HA, Heijnen ML, Wijga A, van Strien RT, Kerkhof M, et al. Respiratory infections in infants: interaction of parental allergy, child care, and siblings- The PIAMA study. Pediatrics. 2001; 108: 943-8.

83. Kramer U, Heinrich J, Wjst M, Wichmann HE. Age of entry to day nursery and allergy in later childhood. Lancet. 1999; 353: 450-4.

84. Leino T, Auranen K, Jokinen J, Leinonen M, Tervonen P, Takala AK. Pneumococcal carriage in children during their first two years: important role of family exposure. Pediatr Infect Dis J. 2001; 20: 1022-7.

85. Louhiala PJ, Jaakkola N, Ruotsalainen R, Jaakkola JJ. Form of day care and respiratory infec- tions among Finnish children. Am J Public Health. 1995; 85: 1109-12.

86. Louhiala PJ, Jaakkola N, Ruotsalainen R, Jaakkola JJ. Day-care centers and diarrhea: a public health perspective. J Pediatr. 1997; 131: 476-9.

87. Lu N, Samuels ME, Shi L, Baker SL, Glover $\mathrm{SH}$, Sanders JM. Child day care risks of common infectious diseases revisited. Child Care Health Dev. 2004; 30: 361-8.

88. Marx J, Osguthorpe JD, Parsons G. Day care and the incidence of otitis media in young children. Otolaryngol Head Neck Surg. 1995; 112: 695-9.

89. Nafstad P, Brunekreef B, Skrondal A, Nystad W. Early respiratory infections, asthma, and allergy: 10-year follow-up of the Oslo Birth Cohort. Pediatrics. 2005; 116: e255-62.

90. Paradise JL, Rockette HE, Colborn DK, Bernard BS, Smith CG, Kurs-Lasky M, et al. Otitis media in 2253 Pittsburgh-area infants: prevalence and risk factors during the first two years of life. Pediatrics. 1997; 99: 318-33.

91. Pass RF, Hutto SC, Reynolds DW, Polhill RB. Increased frequency of cytomegalovirus infection in children in group day care. Pediatrics. 1984; 74: 121-6.

92. Petersson C, Hakansson A. A retrospective study of respiratory tract infections among children in different forms of day care. Scand $\mathbf{J}$ Prim Health Care. 1990; 8: 119-22.

93. - Presser HB. Place of child care and medicated respiratory illness among young American children. J Marriage Fam. 1988; 50: 995-1005.

94. Rasmussen F, Sundelin C. Use of medical care and antibiotics among preschool children in different day care settings. Acta Paediatr Scand. 1990; 79: 838-46.

95. Chellini E, Talassi F, Corbo G, Berti G, De Sario M, Rusconi F, et al. Caratteristiche demografiche, sociali e stili di vita della popolazione infantile in varie aree geografiche italiane. Epidemiol Prev. 2005; 29: 14-23.

96. Ruuska T, Vesikari T. A prospective study of acute diarrhoea in Finnish children from birth to 2 1/2 years of age. Acta Paediatr Scand. 1991; 80: 500-7.

97. Shi L, Lu N, Zhao M, Xu J, Kelly K, Huang Y. Respiratory symptoms and use of medical care 
associated with child day care and health care plan among preschool children. J S C Med Assoc. 2000; 96: 335-40.

98. Silverstein M, Sales AE, Koepsell TD. Health care utilization and expenditures associated with child care attendance: a nationally representative sample. Pediatrics. 2003; 111: e371-5.

99. Ståhlberg M. The influence of form of day care on occurrence of acute respiratory tract infections among young children. Acta Paediatr Scand. 1980; 282(suppl): 1-87.

100. Strangert K. Respiratory illness in preschool children with different forms of day care. Pediatrics. 1976; 57: 191-6.
101. Strangert K. Otitis media in young children in different types of day care. Scand J Infect Dis. 1977; 9: 119-23.

102. Tainio VM, Savilahti E, Salmenpera L, Arjomaa P, Siimes MA, Perheentupa J. Risk factors for infantile recurrent otitis media: atopy but not type of feeding. Pediatr Res. 1988; 23: 509-12.

103. Toroyan T, Roberts I, Oakley A, Laing G, Mugford M, Frost C. Effectiveness of out-of-home day care for disadvantaged families: randomised controlled trial. BMJ. 2003; 327: 906.

104. Wald ER, Guerra N, Byers C. Frequency and severity of infections in day care: three-year follow-up. J Pediatr. 1991; 118: 509-14. 